NASA/TM-2001-210895

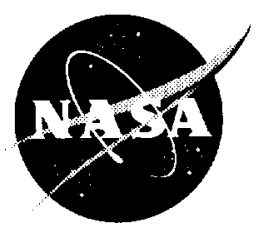

\title{
Demodulation System for Fiber Optic Bragg Grating Dynamic Pressure Sensing
}

John D. Lekki and Grigory Adamovsky

Glenn Research Center, Cleveland, Ohio

Bertram Floyd

Akima, Inc., Brook Park, Ohio

Prepared for the

Eighth Annual International Symposium on Smart Structures and Materials

sponsored by The International Society for Optical Engineering

Newport Beach, California, March 4-8, 2001

National Aeronautics and

Space Administration

Glenn Research Center 
Available from

NASA Center for Aerospace Information 7121 Standard Drive

Hanover, MD 21076
National Technical Information Service 5285 Port Royal Road Springfield, VA 22100

Available electronically at http://gltrs.grc.nasa.gov/GLTRS 


\title{
Demodulation System for Fiber Optic Bragg Grating Dynamic Pressure Sensing
}

\author{
John D. Lekki and Grigory Adamovsky \\ National Aeronautics and Space Administration \\ Glenn Research Center \\ Cleveland, Ohio 44135 \\ Bertram Floyd \\ Akima, Inc. \\ Brook Park, Ohio 44142
}

\begin{abstract}
Fiber optic Bragg gratings have been used for years to measure quasi-static phenomena. In aircraft engine applications there is a need to measure dynamic signals such as variable pressures. In order to monitor these pressures a detection system with broad dynamic range is needed. This paper describes an interferometric demodulator that was developed and optimized for this particular application. The signal to noise ratio was maximized through temporal coherence analysis. The demodulator was incorporated in a laboratory system that simulates conditions to be measured. Several pressure sensor configurations incorporating a fiber optic Bragg grating were also explored. The results of the experiments are reported in this paper.
\end{abstract}

Keywords: Fiber, Sensor, Interferometer, Bragg Grating

\section{Introduction}

Fiber optic sensors are believed to have a significant role to play in future "smart" aerospace vehicles. The benefits of using fiber optic sensors become evident when a large number of sensors are required and size and weight of these sensors are significant issues. In airplanes or launch vehicles the number of sensors desired is increasing and, as always, the size and weight are to be minimized. Low weight is of extreme importance for launch vehicles as the cost for launching each pound into orbit is on the order of several thousand dollars per pound.

An aircraft engine is an excellent candidate for incorporating "smart" capabilities. There are many parameters within an aircraft engine that, if monitored and compensated for. could produce marked improvement in efficiency and reliability. Some examples are liners that detect blade rub damage and compressor blades that detect cracks. The area that is of initial interest for using dynamic pressure measurement is the detection of engine stall or surge conditions. The sensors and sensor demodulation method have been examined with this particular application as the target application.

A sensor capable of detecting stall or surge preconditions could have a significant impact in improving the efficiency of turbomachinery. Presently, turbomachinery is operated at conditions that are outside of a certain margin from stall conditions. In some circumstances operating within this margin, closer to conditions that may induce stall or surge, can increase engine efficiency. Smart aircraft engines capable of detecting stall or surge preconditions, and able to react to cut off such, is proposed as a way to realize these efficiency increases by allowing safe operation with a smaller stall margin $[1,2]$.

It has been shown that stall conditions in turbomachinery are preceded by anomalous pressure fluctuations [3]. In the referenced case for example, the nominal pressure fluctuation in the diffuser due to the passage of compressor blades is approximately $13.8 \mathrm{KPa}(2 \mathrm{psi})$. The stall precursors start at 0.1 seconds before the surge and have peak-to-peak amplitude of approximately $27.6 \mathrm{KPa}$ (4 psi). These numbers are only characteristic of this particular machine. For other machines the location where stall begins and magnitude of precursors would be different. The pressure of the compressor blade wake from this particular machine served as the target pressure sensitivity requirement for the sensor system. The frequencies of blade passage and the frequencies of the stall precursors motivated the target sample frequency. While this application is the preferred objective, sensor configurations were examined that might not be practical for this application but are of interest for dynamic pressure sensing in general. 
The multiplexing capability of the fiber optic Bragg grating and the fact that strain linearly modulates the reflected wavelength are why this sensor type was chosen for this application. The target frequency response was $500 \mathrm{~Hz}$. This narrowed the choices of possible demodulation techniques. As a result, the scanning Fabry-Perot or other scanning demodulation methods were not considered due to their low frequency response.

\section{Theory of Operation}

The demodulation method chosen for the fiber optic Bragg grating sensor is an interferometer, either Michelson or MachZehnder. The chief advantage in a Michelson or Mach-Zehnder interferometer. implemented in a fiber optic or an integrated optic device, is that there is very little optical power lost. An interferometer also has the benefit of having continuous responsivity to wavelength shifts. One disadvantage of an interferometer is the drift in sensitivity due to thermal affects. However, using a feedback loop to keep the output of the interferometer in quadrature can minimize this effect of drift. The techniques to accomplish the compensation can be found elsewhere [4,5].

The output signal from a classical interferometer is a function of a phase difference generated by a difference in optical paths. This phase difference $\phi$ is a product of the optical path difference (OPD) $n \Delta /$ and wavenumber $k$ and can be expressed as

$$
\phi=k n \Delta l
$$

If OPD is kept constant, then the variation in the wavelength would lead to changes in the phase:

$$
d \phi=d k n \Delta l .
$$

Interference of two optical signals with limited coherence length results in a pattern that is influenced not only by the phase difference but also by fringe visibility [5,6,7]. The visibility function, $V$. limits the maximum interferometer response to wavelength shift because the increase in OPD decreases the coherence between the interferometer paths. The visibility function is:

$$
V=\exp \left[-\left(\frac{n \Delta l \Delta \kappa}{4 \sqrt{\ln (2)}}\right)^{2}\right]
$$

where $\Delta \kappa$ is the full width half maximum power of the optical spectrum reflected from the Bragg grating, in wavenumbers. This phase shift and fringe visibility has been used to evaluate optical signals reflected back from fiber optic Bragg gratings [4.5.6]. The current from the photo detector can be expressed as:

$$
i=\rho P V d \phi,
$$

where $\rho$ is photo detector sensitivity,

$P$ is the optical power reflected from the Bragg grating.

Substituting Equations (2) and (3) into Equation (4) gives an equation for the current response as a function of wavelength shift:

$$
i=\rho P n \Delta l d k \exp \left[-\left(\frac{n \Delta l \Delta \kappa}{4 \sqrt{\ln 2}}\right)^{2}\right]
$$

It may be seen from Equation (5) that in order to maximize the sensitivity to wavelength changes, the signal degradation due to coherence loss from an increased $n \Delta l$ must be balanced with the effect of the increased $n \Delta l$ on the phase shift. 
The interferometer output vs. wavelength shift is significantly influenced by the bandwidth of the reflected light from the fiber optic Bragg grating [6]. The full width half maximum (FWHM) power of the optical spectrum for the grating used was experimentally measured to be $0.2 \mathrm{~nm}$ using an optical spectrum analyzer with a resolution of $0.1 \mathrm{~nm}$. The error band of the spectrum analyzer gives a range of FWHM from 0.15 to 0.25 in the measurement of bandwidth. Equation (5) was used to compute the sensitivity to wavelength shift for three different values of FWHM in this range. Results are shown in Figure 1. From the resultant graphs, the optical path difference that will produce an interferometer with maximum sensitivity ranges from $2.5 \mathrm{~mm}$ to $4.2 \mathrm{~mm}$.

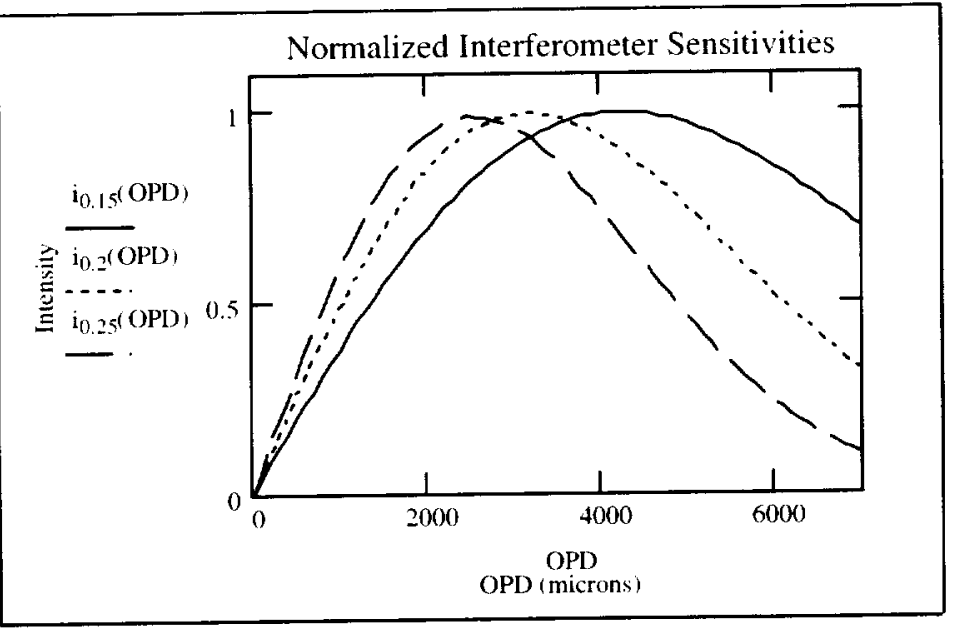

\begin{tabular}{l|l}
\hline$F W H M$ & OPD of Max. Sensitivity \\
\hline $0.15 \mathrm{~nm}$ & $4.2 \mathrm{~mm}$ \\
$0.2 \mathrm{~nm}$ & $3.2 \mathrm{~mm}$ \\
$0.25 \mathrm{~nm}$ & $2.5 \mathrm{~mm}$ \\
\hline
\end{tabular}

Figure 1: Comparison of normalized sensitivities for fiber optic Bragg grating reflected signals with FWHM of $0.15 \mathrm{~nm}, 0.2 \mathrm{~nm}$ and $0.25 \mathrm{~nm}$.

\section{Experiment}

\subsection{Determination of Interferometer Sensitivity}

Figure 2 shows the configuration in which the sensitivity of the interferometer to wavelength shift vs. optical path length difference was determined. The fiber Bragg grating was attached to a rectangular piezoelectric element (PZT) made of Lead Zirconate Titanate that had dimensions of: length $50.8 \mathrm{~mm}$. width $4.69 \mathrm{~mm}$, and thickness $3.43 \mathrm{~mm}$. The fiber was attached parallel to the long dimension. The material used was Navy II type of PZT with $d_{31}$ constant of $185 \times 10^{-12} \mathrm{Volts} / \mathrm{m}$. The applied voltage had a DC component and a time periodic component. The voltage was applied in the direction of thickness. Thus the strain generated in the fiber by the expansion and contraction of the PZT element was mainly along the optical axis of the fiber.

The PZT was driven by a $1 \mathrm{KHz}$ sinusoidal signal with constant amplitude and a zero bias. The translation mirror was used to change the optical path difference $n \Delta l$ in 400 micron increments and a spectrum analyzer measured the (Volt) ${ }^{2}$ Root Mean Squared signal. A fine adjustment of $n \Delta l( \pm \mathrm{I}$ micron) was made to find the local maximum. The maximum response corresponds to the quadrature point. It is this maximum signal that was recorded every 400 microns. This was repeated two times over a range of 8000 microns. The interferometer was taken apart between measurements in order to have two independent measurements. The results from both measurement sets, their average values, as well as a curve calculated from Equation 5 using a bandwidth of $0.2 \mathrm{~nm}$ are shown in Figure 3. The data sets in Figure 3 have been normalized so that the maximum value of optical power reaches unity. This results in a dimensionless normalized intensity response curve as a function of the optical path difference. Despite the fact that only two independent measurement sets were made, the results showed a good correlation between the two sets.

The measured average sensitivity curve in Figure 3(b) shows good agreement to that predicted by Equation (5). The measured average has a peak that does fall within the range $(2.5 \mathrm{~mm}-4.2 \mathrm{~mm})$ predicted from the bandwidth measured. This peak corresponds to the one predicted for a Bragg grating reflection with a $0.16 \mathrm{~nm}$ bandwidth. There is enough difference 
between the measured average and the curve predicted from a $0.2 \mathrm{~nm}$ bandwidth to indicate the importance of measuring an interferometer's responsivity to wavelength shifts. From Figure $3(\mathrm{~b})$. the measured average curve shows a peak at $4 \mathrm{~mm}$ and the calculated peak is at $3.2 \mathrm{~mm}$. If an interferometer were built using the $3.2 \mathrm{~mm} n \Delta /$ then there would be a $14 \%$ reduction in sensitivity as is shown in the comparison of the measured average to the calculated curve.

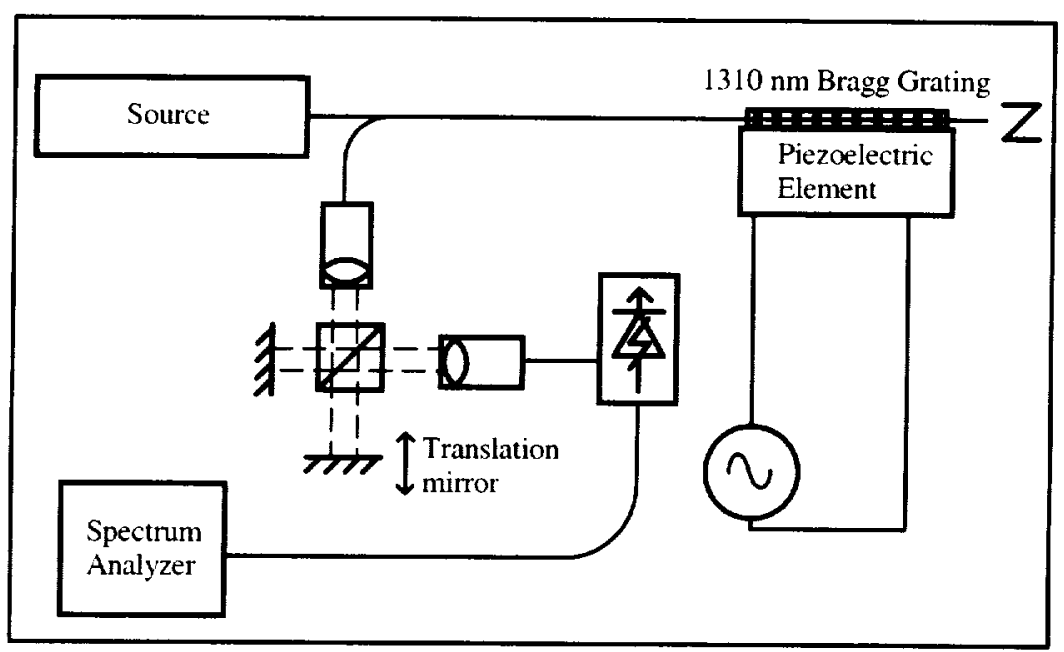

Figure 2: The experimental setup used to determine interferometer sensitivity

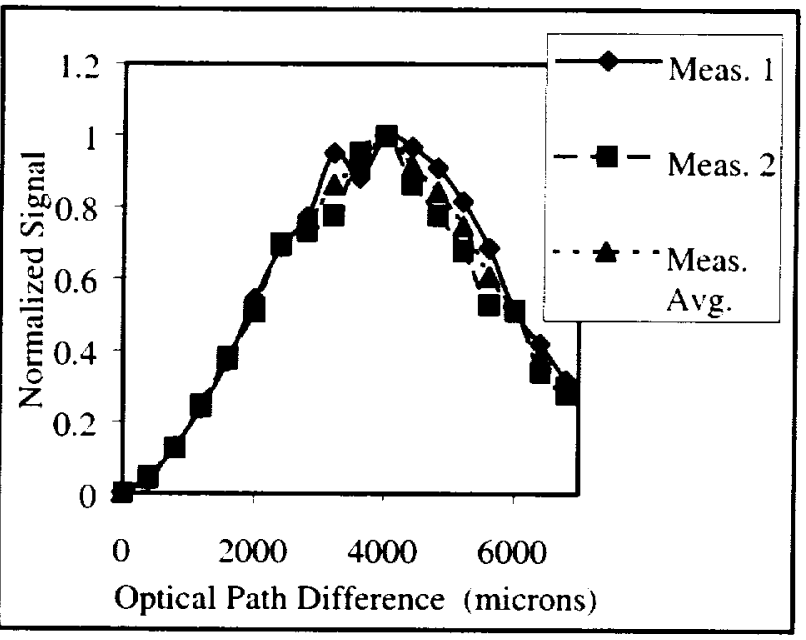

a)

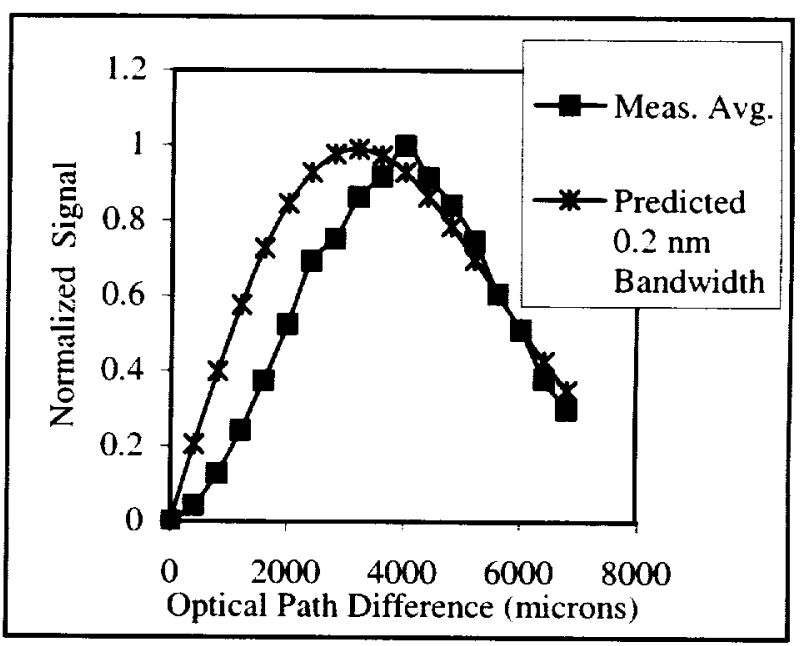

b)

Figure 3: Normalized signals from interferometer as a function of the optical path difference $n \Delta l$ :

a) Experimentally obtained data from two sets of measurements and their average values

b) Comparison of the measured average response and the predicted one for optical signal with $0.2 \mathrm{~nm}$ bandwidth.

The importance of measuring an interferometer to confirm a particular requirement is made apparent from the differences in the predicted curve and measured average curve in Figure 3(b). The measured average peak and the predicted peak have an $n \Delta /$ that is separated by $0.8 \mathrm{~mm}$. The predicted curve in Figure 3 has a value of 0.9 or more over a range of approximately 2000 microns. The range at which the measured average responsivity remains over $0.9 \mathrm{~nm}$ is approximately 900 microns: a range that is less than half that for the predicted curve. At 6000 microns $n \Delta l$, both measured and predicted responsivities were 0.51 . The largest discrepancies between the measured average and predicted curve are observed at lower $n \Delta l$. For instance, at $n \Delta l=2000 \mu \mathrm{m}$ the normalized measured response was 0.52 and the predicted value was 0.85 . 
It was thought that the differences in the predicted curve and the measured average curve might be due to power variability caused from moving the translation mirror away from the beam splitter as shown in Figure 2 . It was decided to measure the power from just the translation mirror path and use this information to compensate the data. The results from measuring this power are shown in Figure 4(a). The optical power does drop off by almost $20 \%$ as the translation mirror is moved away from the beam splitter and so this is a systematic error that should be compensated for. The method to compensate the data comes from the relation of the optical power in the two paths of a Michelson interferometer to the output. The Michelson interferometer's total power can be expressed in terms of the power from the fixed mirror path, $I_{f}$, and the power from the translation mirror path, $I_{i}$, by

$$
I=I_{f}+I_{t}+2 \sqrt{I_{f} I_{t}} \gamma_{t f} \cos (\phi)
$$

where $\gamma_{i f}$ is the complex degree of coherence and $\phi$ is the phase difference of the light from the fixed path and the translation path [4].

The complex degree of coherence, $\gamma_{f}$, decreases from one as the paths become imbalanced, so for all points other than zero, the third term of Equation (6) cannot be as large as the first two. The first two terms of Equation (6) represent the DC level upon which the power due to phase modulation oscillates. Since only the dynamic component is important only the third term needs to be considered. In a normalized response the intensity is compensated for by

$$
I_{c}(x)=2 \sqrt{I_{f} I_{t}(x)} \gamma_{t f} \cos (\phi) \sqrt{\frac{I_{t \max }}{I_{t}(x)}},
$$

where $I_{\max }$ is the maximum optical power from the translation mirror, and $I_{t}(x)$ is the optical power measured at each translation point.

In Figure 4 (b) each of the measured data points has been corrected for power variations by multiplying the measured sensitivity by a correction factor equal to the square root of the maximum power measured from the translation mirror divided by the measured power value of each position. The correction in general only amounted to one percentage point correction, and did not change the location of the measured average peak sensitivity.

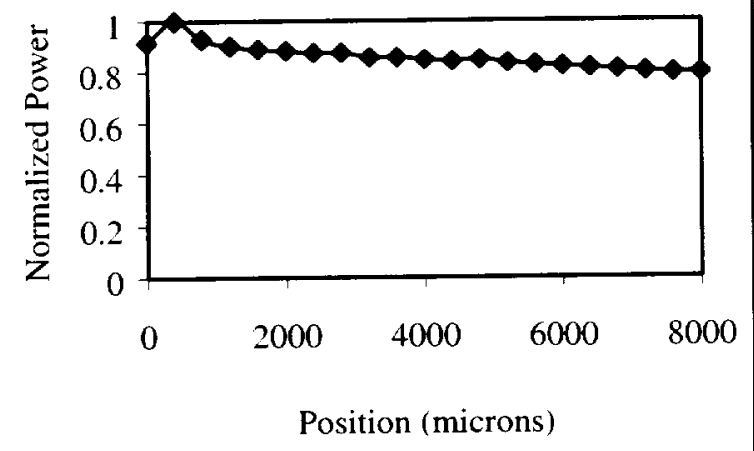

a)

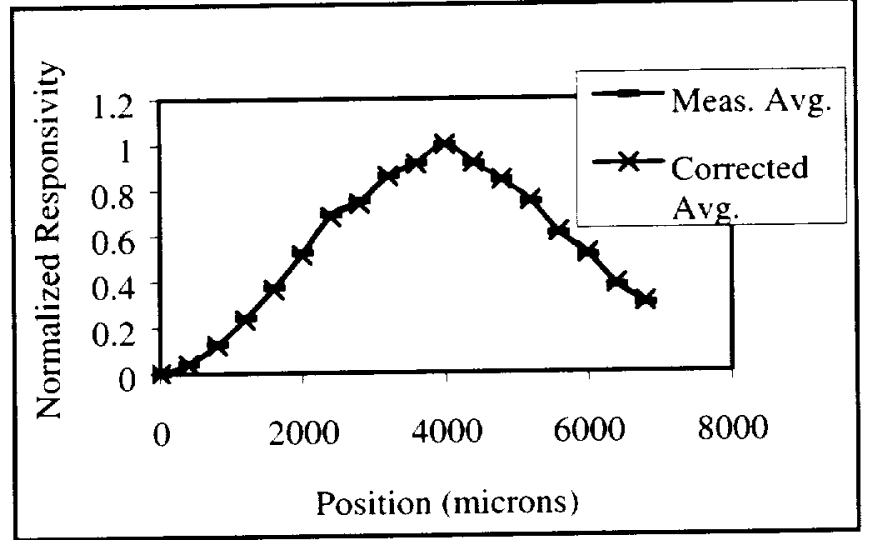

b)

Figure 4: Compensation for loss of reflected power in translation mirror path:

a) The optical power from the translation mirror path of the interferometer as a function of position.

b) The responsivity data corrected for translation mirror power variance.

\subsection{Sensor Operation with imposed strain}

In Figure 5 the output from the fiber optic sensor system is shown with a $5 \mathrm{~Hz}$ ramp function applied to the PZT. The dynamic voltage was $38 \mathrm{v}_{\mathrm{p}-\mathrm{p}}$ and there was also a $100 \mathrm{~V} \mathrm{DC}$ offset applied to the PZT. A straight thin line has been added for 
comparison of linearity. The slightly nonlinear response is believed to be a second order effect, which becomes significant with large amplitude strain. This should not be a problem for pressure sensing as the strains in the fiber are expected to be of a low magnitude.

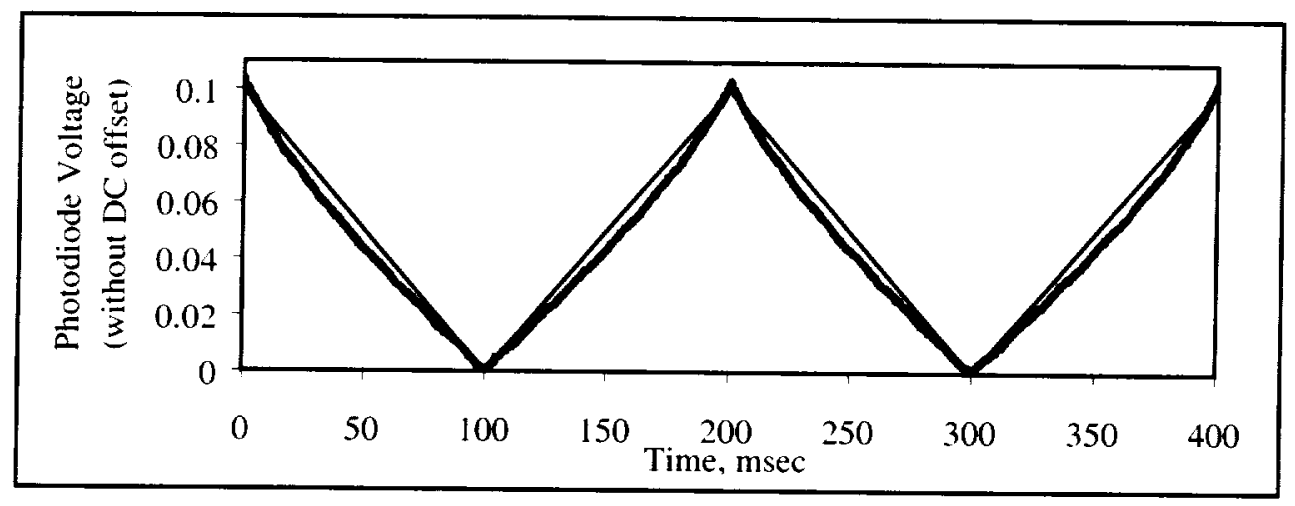

Figure 5: The Michelson demodulated signal from piezoelectric strain. A thin straight line is added as a reference.

\subsection{Tests of the Sensor with flow imposed strain}

Since future possible applications are as an element of a pressure sensor the Bragg grating was tested in three configurations that are shown in Figure 6. In each configuration the strain was imposed by airflow from a pressurized nozzle that was chopped with a frequency of $10 \mathrm{~Hz}$ by an optical chopper wheel. To monitor the total pressure from the jet a pressure transducer was placed directly behind the fiber. In the first configuration, Figure $6(a)$, the fiber optic Bragg grating is held in tension within the flow field. The second configuration, Figure $6(\mathrm{~b})$, is similar to the first, the fiber was held at tension within the flow but the distance between the anchor points was decreased. In the third configuration. Figure 6(c), the fiber is attached to a flexible membrane but in all other respects is the same as in the second configuration. This membrane offers a larger area exposed to the flow so the fiber is strained more from the chopped flow.

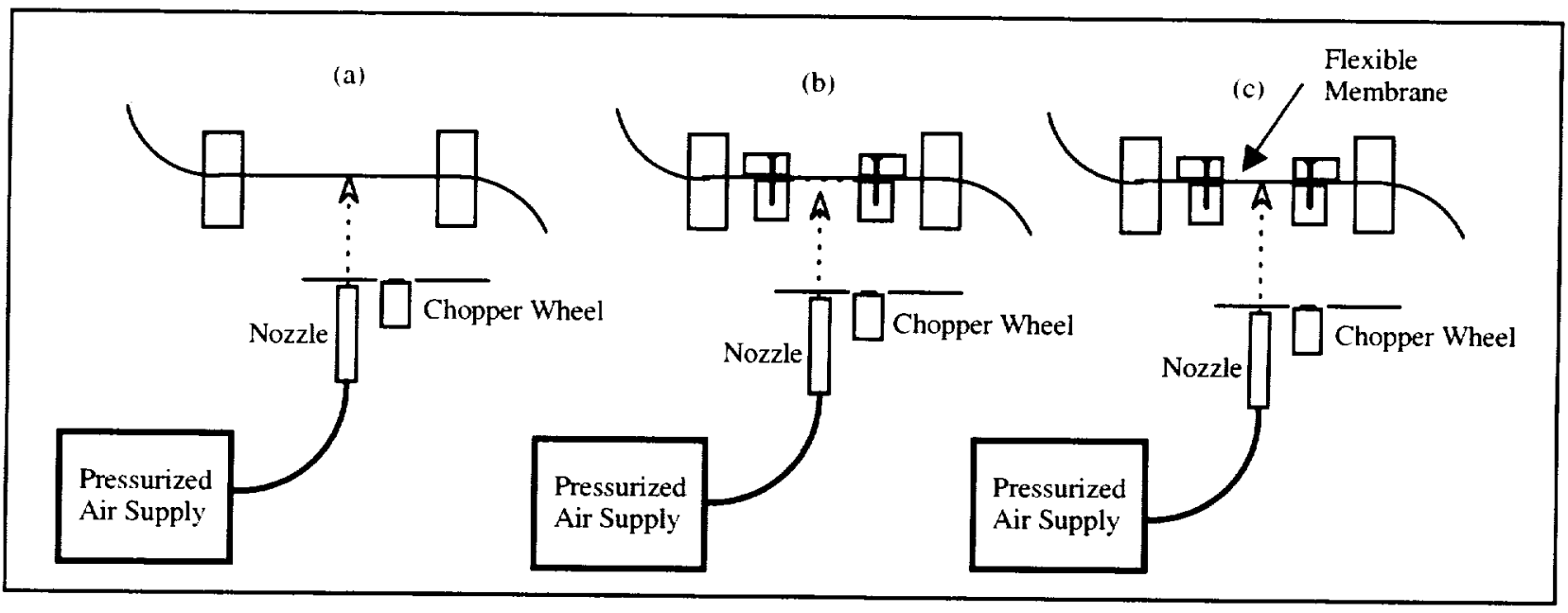

Figure 6: Three sensor configurations tested:

a) Fiber held at tension between two holders with pressurized air causing pressure variations,

b) Fiber held at tension between two holders with shorter distance between anchor points,

c) Fiber attached to back of cylinder with flexible membrane. 
In all three configurations the fiber was subjected to periodic flow variations. The oscillatory component of total pressure was about $1.75 \mathrm{KPa}$ and the frequency $10 \mathrm{~Hz}$. Figure 7 demonstrates the pressure variations detected by the pressure transducer installed behind the Bragg grating.

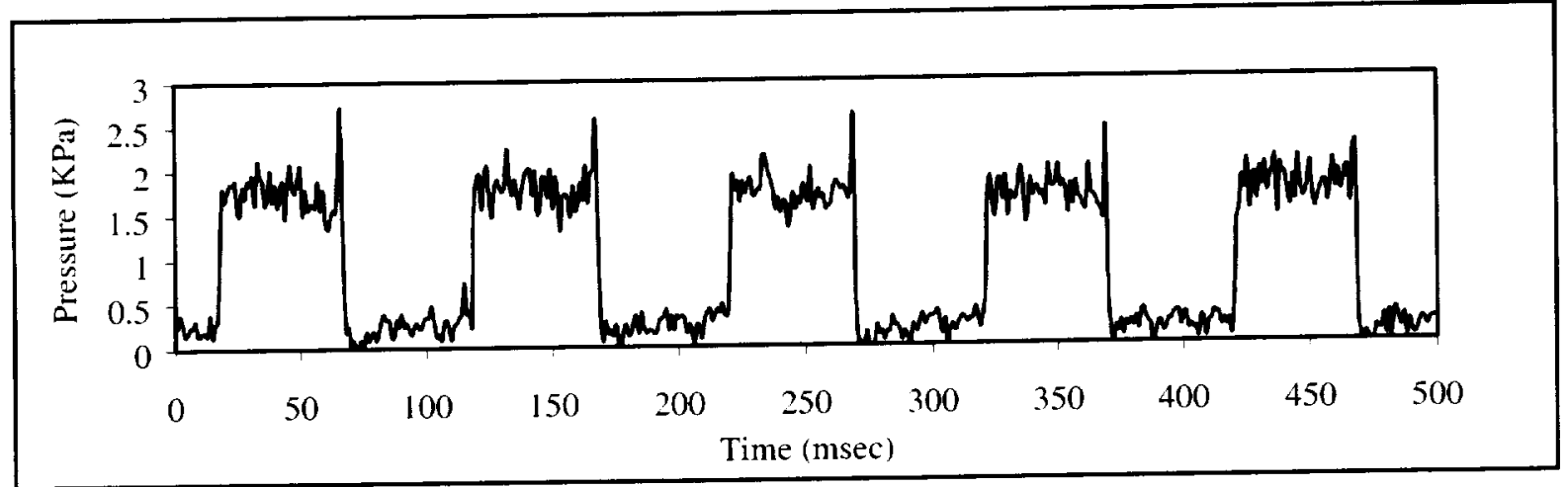

Figure 7: Typical signal from the pressure transducer.

In these experiments signals from the interferometer were processed by a signal processing unit which replaced the spectrum analyzer in the setup described in Figure 2. The signal processing involves ratioing of a reference signal by the filtered signal from the interferometer. This makes the resultant signal less sensitive to intensity variations that occur in connecting optical cables. These processed signals are dimensionless. Figure 8 contains displays of the processed signals obtained from the three fiber configurations in Figure 6.

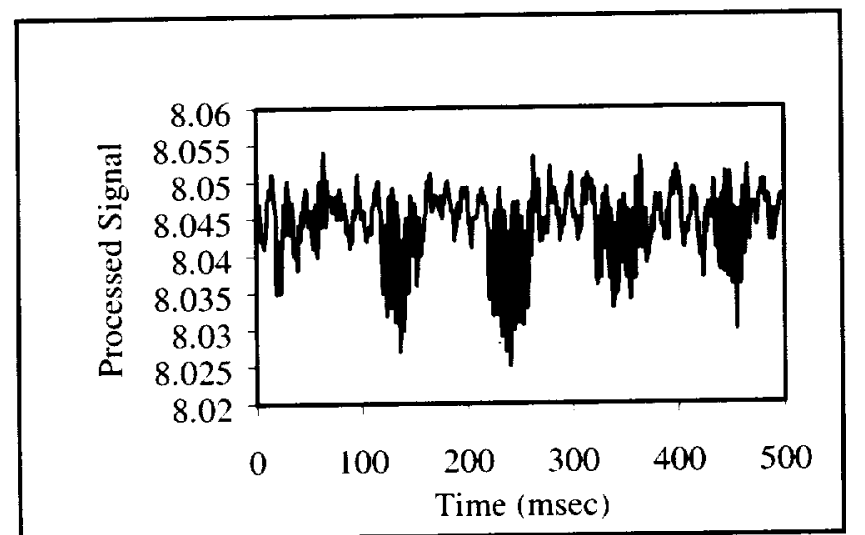

a)

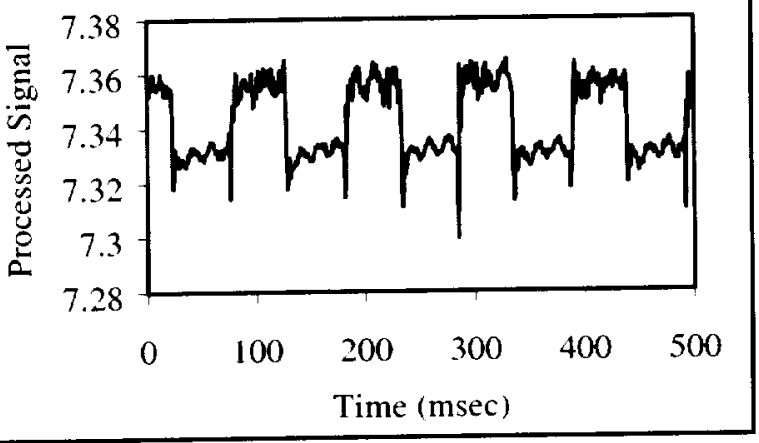

b)

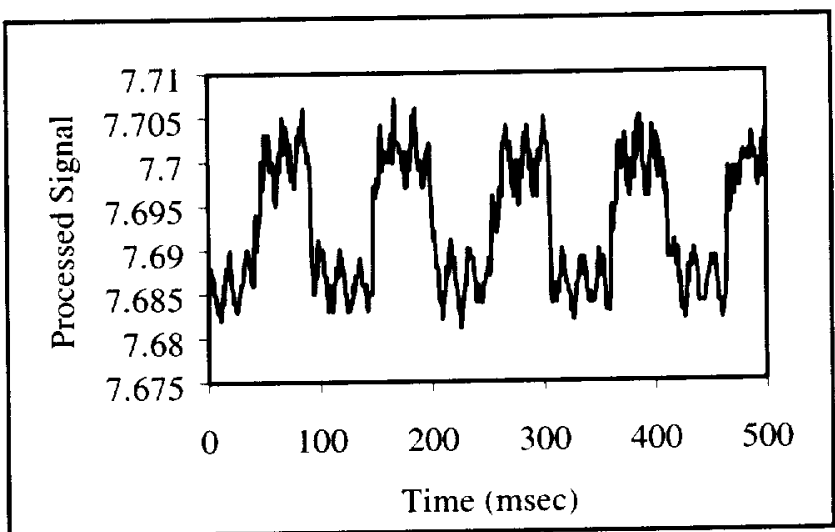

c)

Figure 8: Processed signals obtained under pressure variations from Figure 7 for the three sensor configurations: 
a) Data for sensor configuration described in Figure 6(a);

b) Data for sensor configuration described in Figure 6(b):

c) Data for sensor configuration described in Figure 6(c).

Analysis of the data obtained has shown that all three sensor configurations may be able to detect the total pressure fluctuations necessary. The second and third configurations of the sensor possess the best response to pressure pulses as is shown in Figure $8(b, c)$. The frequency response in both configurations was nominally the same as that of the pressure transducer.

Higher frequency total pressure pulses have also been applied in order to examine the capability to sense higher frequencies. A fiber set up in the second configuration has been preliminarily tested at frequencies up to 200 hundred Hertz with a different nozzle geometry. Results are shown in Figure 9.

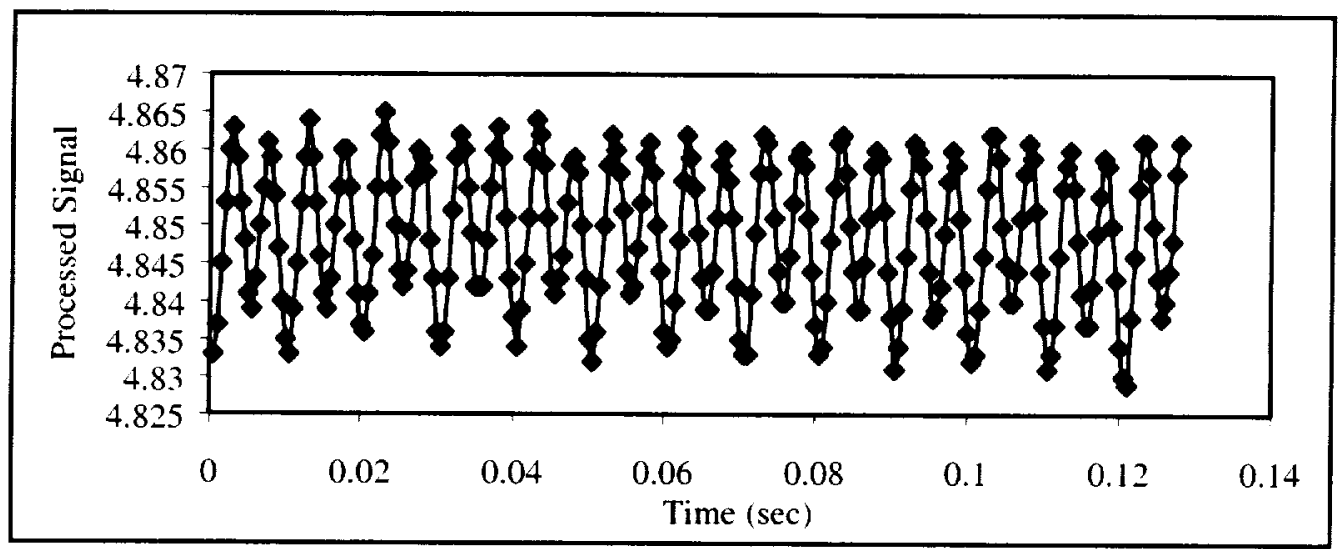

Figure 9. Processed signal from interferometer detecting $200 \mathrm{~Hz}$ pressure variations.

\section{DISCUSSION}

The Michelson and Mach-Zehnder interferometers have known capabilities for fiber optic sensor demodulation. Their sensitivity to wavelength shift has been previously shown to be a function of the optical path length difference $n \Delta l$. However, these interferometers are difficult to design for this application without first measuring their responsivity curve. This is because the error in the measurement of the fiber Bragg grating's spectral bandwidth places the $n \Delta l$ in a range of values where the responsivity will vary as much as $14 \%$.

The interferometer has been shown capable of demodulating slight wavelength shifts caused from pressure fluctuations straining a fiber within a flow field. The high sensitivity coupled with the ability to detect frequencies of the order of few hundred Hertz would permit detection of pressure waves associated with stall precursors in the referenced turbomachinery. The problem of how to configure the sensor inside a turbomachine does remain. The first and second configuration of the fiber in Figure $6(a, b)$ would be the least intrusive but may not survive for long if there is contamination within the airflow, such as sand that is sucked into an aircraft engine. The third configuration. Figure 6 (c) simulates a configuration where the fiber is built into a sensor housing that would be installed within an engine. However a sensing device based on this principle would be a bulky one that would be more difficult to install and may have an adverse effect on the airflow within the engine. If the problem of how to install a fiber within a turbomachine can be solved, it appears that an interferometer would have the sensitivity necessary to demodulate the Bragg grating wavelength shifts. To achieve the goal of using fiber optic Bragg gratings to detect stall precursors, future development will be focused on sensor configurations including the use of Bragg gratings with axially selective sensitivity and sensor housings to secure these gratings.

\section{REFERENCES}

1. I. J. Day. "Active Suppression of Rotating Stall and Surge in Axial Compressors," Joumal of Turbomachinery, 115, pp. 40-47. 1993.

2. A. H. Epstein, J. E. Ffowcs Williams, and E. M. Greitzer, "Active Suppression of Aerodynamic Instabilities in Turbomachines", AIAA Journal of Propulsion and Power, 5, pp. 204-211, 1986. 
3. M. Wernet and M. Bright, "Dissection of Surge in a High Speed Centrifugal Compressor using Digital PIV", $37^{\text {th }}$ AIAA Aerospace Sciences Meeting, Reno, 1999.

4. M. Song. S. Yin, and P. Ruffin. "Fiber Bragg grating strain sensor demodulation with quadrature sampling of a MachZehnder interferometer," Applied Optics, 39. pp. 1106-1111 (2000).

5. A. D. Kersey, T. A. Berkoff. and W. W. Morey, "High-Resolution Fibre-Grating Based Strain Sensor with Interferometric Wavelength-Shift Detection." Electronics Letters, 28, pp. 236-238 (1992).

6. R. S. Weis, A. D. Kersey, and T. A. Berkoff, "A Four-Element Fiber Grating Sensor Array with Phase-Sensitive Detection," IEEE Photonics Letters, 6, pp. 1469-1472 (1994).

7. M. Born and E. Wolf, Principles of Optics, Ch. 10, Pergamon Press, Oxford, 1980.

8. J. R. Dunphy, G. Meltz, and W. W. Morey, "Optical Fiber Bragg Gratin Sensors: A Candidate for Smart Structure Applications." Fiber Optic Smart Structures, Eric Udd. pp. 271-286. Wiley-Interscience. New York, 1995. 
Public reporting burden for this collection of information is estimated to average 1 hour per response, including the time for reviewing instructions, searching existing data sources, gathering and maintaining the data needed, and completing and reviewing the collection of information. Send comments regarding this burden estimate or any other aspect of this collection of information, including suggestions for reducing this burden, to Washington Headquarters Services. Directorate for Information Operations and Reports, 1215 Jefferson Davis Highway. Suite 1204. Arlington, VA 22202-4302, and to the Ottice of Management and Budget. Papenwork Reduction Project (0704-0188), Washington, DC 20503.

\begin{tabular}{l|l|l|} 
1. AGENCY USE ONLY (Leave blank) & 2. REPORT DATE & 3. REPORT TYPE AND DATES COVERED
\end{tabular}

4. TITLE AND SUBTITLE

August 2001

Technical Memorandum

5. FUNDING NUMBERS

Demodulation System for Fiber Optic Bragg Grating Dynamic Pressure Sensing

6. AUTHOR(S)

WU-704-30-53-00

John D. Lekki, Grigory Adamovsky. and Bertram Floyd

7. PERFORMING ORGANIZATION NAME(S) AND ADDRESS(ES)

National Aeronautics and Space Administration

John H. Glenn Research Center at Lewis Field

Cleveland, Ohio 44135-3191

8. PERFORMING ORGANIZATION REPORT NUMBER

9. SPONSORING/MONITORING AGENCY NAME(S) AND ADDRESS(ES)

National Aeronautics and Space Administration

Washington. DC 20546-0001

10. SPONSORINGMONITORING AGENCY REPORT NUMBER

NASA TM-2001-210895

\section{SUPPLEMENTARY NOTES}

Prepared for the Eighth Annual International Symposium on Smart Structures and Materials sponsored by The International Society for Optical Engineering, Newport Beach. California, March 4-8, 2001. John D. Lekki and Grigory Adamovsky, NASA Glenn Research Center; and Bertram Floyd, Akima, Inc., 2001 Aerospace Parkway, Brook Park. Ohio 44142. Responsible person. John Lekki, organization code 5520. 216-433-5650.

12a. DISTRIBUTION/AVAILABILITY STATEMENT

12b. DISTRIBUTION CODE

Unclassified - Unlimited

Subject Categories: 06, 19, and 35

Distribution: Nonstandard

Available electronically at http://gltrs.grc,nasa.gov/GLTRS

This publication is available from the NASA Center for AeroSpace Information. 301-621-0390.

13. ABSTRACT (Maximum 200 words)

Fiber optic Bragg gratings have been used for years to measure quasi-static phenomena. In aircraft engine applications there is a need to measure dynamic signals such as variable pressures. In order to monitor these pressures a detection system with broad dynamic range is needed. This paper describes an interferometric demodulator that was developed and optimized for this particular application. The signal to noise ratio was maximized through temporal coherence analysis. The demodulator was incorporated in a laboratory system that simulates conditions to be measured. Several pressure sensor configurations incorporating a fiber optic Bragg grating were also explored. The results of the experiments are reported in this paper.

\section{SUBJECT TERMS}

Sensors: Optical sensors; Fiber optic Bragg gratings; Fiber; Interferometer

\begin{tabular}{|c|c|c|}
\hline $\begin{array}{c}\text { 17. SECURITY CLASSIFICATION } \\
\text { OF REPORT } \\
\text { Unclassified }\end{array}$ & $\begin{array}{c}\text { 18. SECURITY CLASSIFICATION } \\
\text { OF THIS PAGE } \\
\text { Unclassified }\end{array}$ & $\begin{array}{c}\text { 19. SECUAITY CLASSIFICATION } \\
\text { OF ABSTRACT } \\
\text { Unclassified }\end{array}$ \\
\hline
\end{tabular}

NSN 7540-01-280-5500 


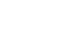


